\title{
A naturally occurring variation in the BrMAM-3 gene is associated with aliphatic glucosinolate accumulation in Brassica rapa leaves
}

\author{
Jifang Zhang ${ }^{1,2}$, Hui Wang ${ }^{1,3}$, Zhiyuan Liu', Jianli Liang ${ }^{1}$, Jian Wu' ${ }^{1}$, Feng Cheng ${ }^{1}$, Shiyong Mei $^{2}$ and Xiaowu Wang ${ }^{1}$
}

\begin{abstract}
Glucosinolate profiles significantly vary among Brassica rapa genotypes. However, the molecular basis of these variations is largely unknown. In this study, we investigated a major quantitative trait locus (QTL) controlling aliphatic glucosinolate accumulation in B. rapa leaves. The QTL, which encompasses three tandem MAM genes and two MYB genes, was detected in two $\mathrm{BC}_{2} \mathrm{DH}$ populations. Among the five-candidate genes, only the expression level of BrMAM3 (Bra013007) was significantly correlated with the accumulation of aliphatic glucosinolates in B. rapa leaves. We identified a naturally occurring insertion within exon 1 of BrMAM-3, which is predicted to be a loss-of-function mutation, as confirmed by qRT-PCR. We determined that the loss of function was associated with the low glucosinolate content in B. rapa accessions. Furthermore, overexpressing the BrMAM-3 gene resulted in an increase in total aliphatic glucosinolates in Arabidopsis transgenic lines. Our study provides insights into the molecular mechanism underlying the accumulation of aliphatic glucosinolates in B. rapa leaves, thereby facilitating in the manipulation of total aliphatic glucosinolate content in Brassica crops.
\end{abstract}

\section{Introduction}

Glucosinolates are sulfur- and nitrogen-containing plant secondary metabolites that commonly occur in the order Brassicales, including important Brassica crops such as oilseed rape (B. napus), cabbage (B. oleracea, Capitata group), and broccoli (B. oleracea, Italica group), and the model plant Arabidopsis thaliana. Physical tissue or cell injury causes these amino acid-derived thioglycosides to cooccur with specific $\beta$-glucosidases called myrosinases and associated proteins, thereby generating an activated plant defense system known as the "mustard oil bomb" ${ }^{\text {". Gluco- }}$ sinolate derivatives such as isothiocyanates, thiocyanates,

\footnotetext{
Correspondence: Xiaowu Wang (wangxiaowu@caas.cn)

${ }^{1}$ Institute of Vegetables and Flowers, Chinese Academy of Agricultural

Sciences, 100081 Beijing, China

${ }^{2}$ Institute of Southern Economic Crops, Institute of Bast Fiber Crops, Chinese

Academy of Agricultural Sciences, 410205 Changsha, China

Full list of author information is available at the end of the article.

These authors contributed equally: Jifang Zhang, Hui Wang
}

and nitriles have a wide range of biological functions, which include anti-carcinogenicity in humans ${ }^{2-6}$, anti-nutritional effects using seed meal in animals ${ }^{7}$, and insect pest repellent and fungal disease suppression ${ }^{8,9}$. Moreover, glucosinolates are responsible for the special flavors of Brassica vegetables such as $B$. rapa and $B$. oleacrea ${ }^{10,11}$. Due to their diverse roles in plant metabolism, animal nutrition, disease, and flavors, glucosinolates are a potential target for genetic manipulation and applications in crop improvement programs.

Glucosinolates are classified into aliphatic, aromatic, and indole glucosinolates, depending on their precursor amino acids ${ }^{12,13}$. Glucosinolate biosynthesis occurs in three independent stages: chain elongation of the precursor amino acid, formation of the core structure, and side-chain modifications. In A. thaliana, chain elongation is an iterative three-step process that operates predominantly on Met and results in up to six methylene

\section{(c) The Author(s) 2018}

(c) (i) Open Access This article is licensed under a Creative Commons Attribution 4.0 International License, which permits use, sharing, adaptation, distribution and reproduction in any medium or format, as long as you give appropriate credit to the original author(s) and the source, provide a link to the Creative Commons license, and indicate if changes were made. The images or other third party material in this article are included in the article's Creative Commons license, unless indicated otherwise in a credit line to the material. If material is not included in the article's Creative Commons license and your intended use is not permitted by statutory regulation or exceeds the permitted use, you will need to obtain permission directly from the copyright holder. To view a copy of this license, visit http://creativecommons.org/licenses/by/4.0/. 
groups, which contribute to the variations in glucosinolate structures. The committed step during Met side-chain elongation is catalyzed by methylthioalkylmalate synthase $(\mathrm{MAM})^{12,14-16}$, which is derived from isopropylmalate synthase (IPMS) of Leu biosynthesis. ${ }^{17}$ Recent reports have confirmed that glucosinolate levels are controlled by at least six R2R3-MYB superfamily transcription factors $^{18-23}$. In $A$. thaliana, the aliphatic glucosinolates genes are regulated by AtMYB28, AtMYB29, and AtMYB76 genes $^{18-22}$, whereas AtMYB34, AtMYB51, and AtMYB122 control the formation of indole glucosinolates ${ }^{23}$. Additionally, MYC2, MYC3, and MYC4 regulate glucosinolate biosynthesis by directly interacting with glucosinolaterelated $\mathrm{MYBs}^{24}$.

Brassica crops are of great economic importance to human beings because these are a rich source of beneficial health glucosinolates such as glucoraphanin and sulforaphane. Vegetable forms of B. rapa (Chinese cabbage, turnip, pakchoi, komatsuna, mizuna green, and rapini) are widely cultivated in many parts of the world ${ }^{25}$, and individual plants generally contain a limited number of major aliphatic glucosinolate profiles ${ }^{10,26}$. Glucosinolate content and profiles are highly variable and accession-specific in various $B$. rapa genotypes, in which the aliphatic (4C) 3butenyl and the aliphatic (5C) 4-pentenyl glucosinolates are the predominant glucosinolates. To date, 102 putative genes of the glucosinolate biosynthesis pathway of $B$. rapa have been inferred by comparative genomic analyses ${ }^{27}$. The expression of seven MYB transcription factors in different organs of Chinese cabbage (B. rapa ssp. Pekinensis) has been investigated ${ }^{28}$. The expression profiles of BrMYB28 and BrMYB29 in stems differ from those in other organs ${ }^{28}$. In addition, three genes encoding AOP2 are differentially expressed in $B$. $\mathrm{rapa}^{29}$. However, more work is required to characterize the genes involved in glucosinolate biosynthesis in B. rapa.

The level and composition of aliphatic glucosinolates are under complex genetic control and are highly heritable ${ }^{30}$. Quantitative trait locus (QTL) analysis is a powerful method to study the genetics underpinning quantitative variations in glucosinolate profiles and to estimate the number of variable loci affecting a trait. ${ }^{31}$ QTL analysis of seed and leaf glucosinolates has been conducted in $A$. thaliana $a^{9,14,32,33}, B$. oleracea $^{34}, B$. napus $^{35-37}$, B. juncea ${ }^{38,39}$, as well as B. rapa ${ }^{26}$. In Arabidopsis, the epistatic interaction of two major genetic QTL controlling total aliphatic glucosinolates that map to the GS-Elong (MAM1 and MAM-L) and GS-AOP loci regulate aliphatic glucosinolate accumulation ${ }^{14}$. In B. rapa, QTL analysis has identified 16 loci that control aliphatic glucosinolate accumulation, three loci that regulate total indolic glucosinolate concentration, and three loci that influence aromatic glucosinolate concentrations ${ }^{26}$. Although these reports have identified QTLs that control the variability of glucosinolate contents and profiles across Brassica species, its underlying molecular genetic mechanism remains unclear.

Genome polyploidization is an evolutionary process that fuels diversity in plant species ${ }^{40}$. Besides ancient wholegenome duplication events involving ancestral Arabidopsis and Brassica species ${ }^{41}$, Brassica crops underwent additional whole-genome triplication (WGT) events. These genome duplication events followed by gene losses during rediploidization resulted in highly complex relationships among the regulatory pathways of aliphatic glucosinolate biosynthesis in Brassica species compared to that in Arabidopsis. The $M A M$ genes are often found as clusters of tandem arrays and differentiated in the Arabidopsis and A. lyrata genomes $^{9,12}$. In $B$. rapa, seven $M A M$ genes, comprising five syntenic and two non-syntenic, have been identified ${ }^{27}$; however, their function in relation to glucosinolate accumulation is poorly understood.

In our previous study, we analyzed the phylogenetic and syntenic relationships of $M A M$ genes from 13 sequenced Brassicaceae species. Based on these analyses, we proposed that the syntenic loci of $M A M$ genes underwent lineage-specific evolution routes and were driven by positive selection after the divergence from Aethionema arabicum $^{29}$. Upon the divergence of the Brassica genus, $B$. rapa retained five syntenic $M A M$ genes that were generated via WGT followed by biased gene loss. BrMAM-1 and $B r M A M-2$ are clustered in the medium-fractionated subgenome (MF1), whereas BrMAM-3, BrMAM-4, and $B r M A M-5$ are clustered in the least-fractionated subgenome (LF). Furthermore, BrMAM-3 and BrMAM-1/2 are homologous genes. However, the contribution of these $M A M$ genes in the observed variations in aliphatic glucosinolate accumulation of $B$. rapa remains unclear.

In this study, we investigated a major QTL locus controlling the accumulation of aliphatic glucosinolates in $B$. rapa leaves. The QTL locus includes a complex loci with three tandem $M A M$ genes (BrMAM-3, BrMAM-4, and $B r M A M-5)$ and a nearby loci with two $M Y B$ genes (BrMYB28.1 and BrMYB34.1). All these genes are possibly involved in controlling aliphatic glucosinolate accumulation in B. rapa. The present study was conducted to clarify the gene(s) contributing to the significant QTLs involved in glucosinolate composition and accumulation in $B$. rapa leaves. Our study may facilitate the genetic engineering of plants to accumulate glucosinolates without compromising overall plant fitness.

\section{Results}

Expression of BrMAM-3 is significantly associated with the accumulation of aliphatic glucosinolates

A major QTL locus on chromosome 3 was consistently detected by MapQTL4 in the RC_BC ${ }_{2} \mathrm{DH}$ and $\mathrm{YS}_{-} \mathrm{BC}_{2} \mathrm{DH}$ populations, which explained a large proportion $(31.0 \%$ 


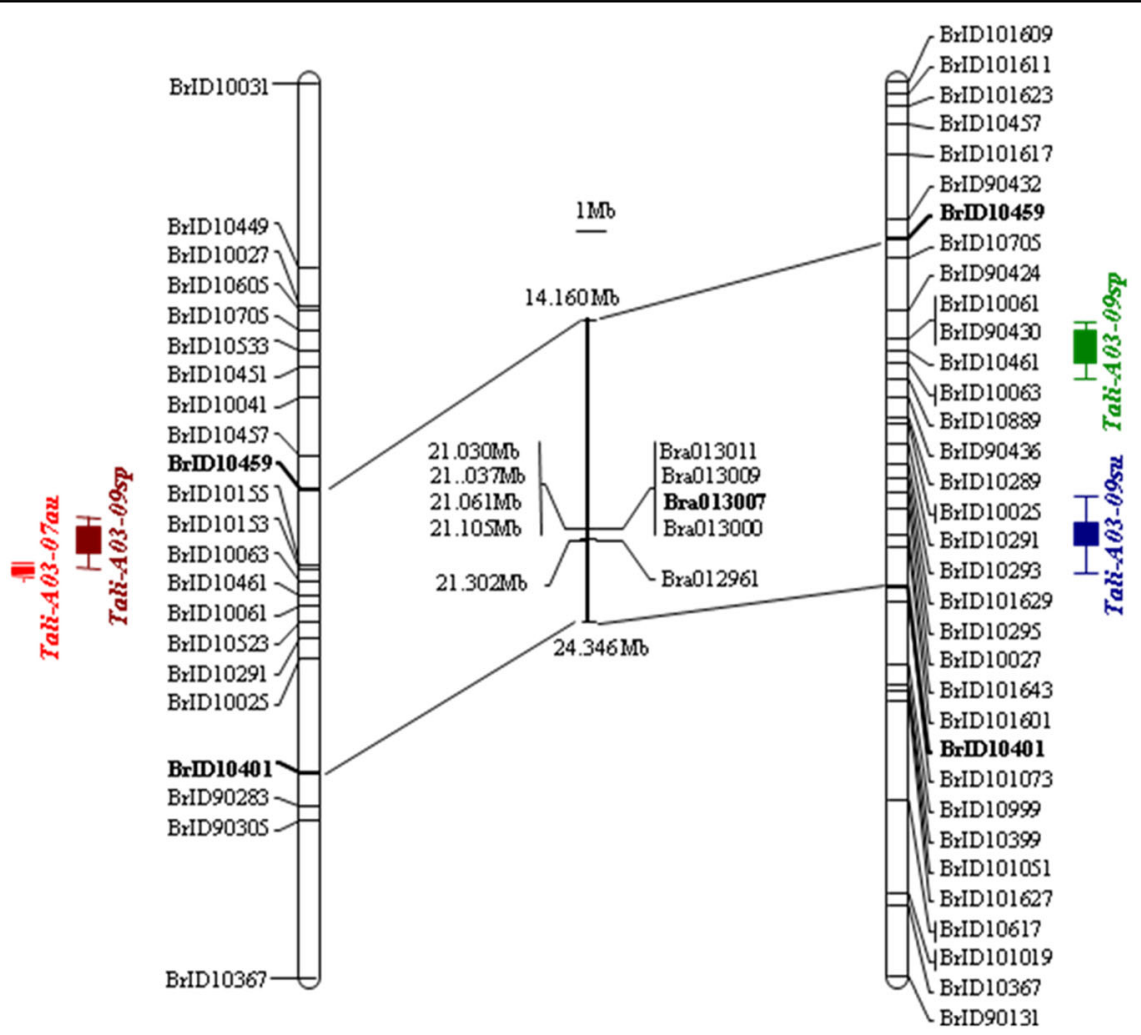

Fig. 1 A major QTL locus on chromosome 3. A major QTL was mapped to a 10.185-Mb genomic DNA region between markers BrID10459 and BrlD10401 in RC_BC 2 DH (a) and YS_BC_ 2 DH (b) populations in autumn of 2007 and the spring of 2009, and in the spring of 2009 and the summer of 2009, respectively

Table 1 Correlation analysis between expression of candidate genes and dominant glucosinolates in Brassica rapa

\begin{tabular}{|c|c|c|c|c|c|c|c|c|c|}
\hline Genes & PRO & NAP & GBN & $40 \mathrm{OH}$ & GBC & NAS & $4 \mathrm{ME}$ & NEO & SUM \\
\hline BrMYB28.1 (Bra012961) & -0.06 & 0.25 & 0.12 & 0.06 & 0.10 & 0.04 & 0.28 & 0.03 & 0.25 \\
\hline$P$ value & 0.66 & 0.07 & 0.41 & 0.68 & 0.47 & 0.80 & 0.04 & 0.86 & 0.08 \\
\hline BrMYB34.1 (Bra13000) & -0.10 & 0.42 & 0.21 & 0.46 & 0.06 & 0.13 & -0.09 & 0.00 & 0.40 \\
\hline$P$ value & 0.46 & 0.00 & 0.14 & 0.00 & 0.66 & 0.37 & 0.52 & 1.00 & 0.00 \\
\hline BrMAM-3 (Bra013007) & -0.13 & 0.70 & 0.07 & 0.43 & 0.01 & 0.03 & -0.14 & -0.06 & 0.57 \\
\hline$P$ value & 0.34 & $<0.0001$ & 0.62 & 0.00 & 0.96 & 0.83 & 0.34 & 0.70 & $<0.0001$ \\
\hline BrMAM-4 (Bra013009) & -0.06 & -0.14 & 0.24 & -0.12 & -0.01 & 0.07 & -0.04 & 0.16 & -0.03 \\
\hline$P$ value & 0.68 & 0.32 & 0.08 & 0.39 & 0.96 & 0.63 & 0.77 & 0.27 & 0.81 \\
\hline BrMAM-5 (Bra013011) & -0.11 & 0.13 & 0.09 & 0.44 & 0.47 & 0.24 & 0.02 & 0.10 & 0.16 \\
\hline$P$ value & 0.43 & 0.36 & 0.53 & 0.00 & 0.00 & 0.08 & 0.88 & 0.48 & 0.27 \\
\hline
\end{tabular}

and $53.0 \%$, respectively, in autumn of 2007 and spring of $2009 ; 29.0 \%$ and $12.9 \%$, respectively, in spring of 2009 and summer of 2009) of the observed phenotypic variations in total aliphatic glucosinolates (Fig. 1). There are eight genes (Supplementary Table S1, S2) in the major QTL region and five were considered as candidate genes, including three tandem $M A M$ genes, namely, $B r M A M-3$ (Bra013007), BrMAM-4 (Bra013009), and BrMAM-5
(Bra013011), and two MYB genes, namely, BrMYB34 (Bra013000) and BrMYB28 (Bra012961).

The association between the expression of candidate genes (Supplementary Table S3) and the glucosinolate profiles was analyzed in 52 double-haploid (DH) lines (Table 1). The profiles of eight glucosinolates, including three aliphatic,3-butenyl- (NAP), 4-pentenyl- (GBN), and 2-hydroxy-3-butenyl-glucosinolate (PRO), four indolic3- 


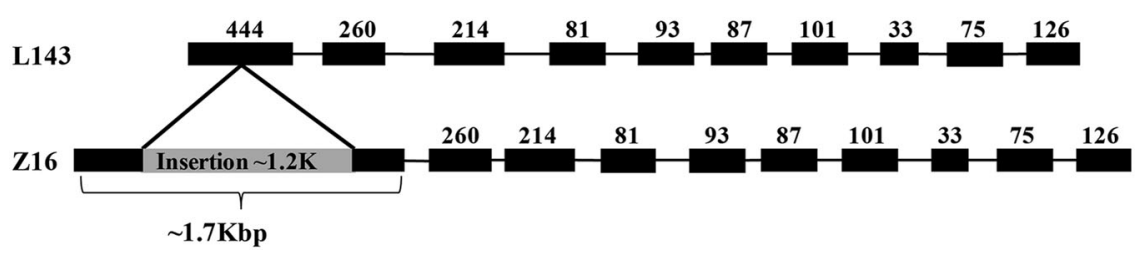

Fig. 2 Gene structure of BrMAM-3 in accessions L143 and Z16. Exons are shown in black blocks and numbers above the blocks indicate exon length. The gray block in exon one indicates the transposon insertion

indolmethyl- (GBC), 1-methoxy-3-indoylmethyl- (NEO), 4-hydroxy-3-indoylmethyl- (4OH), and 4-methoxy-3indoylmethyl-glucosinolate (4ME), and one aromatic glucosinolate, 2-phenylethyl-glucosinolate (NAS), were detected in all varieties. The total glucosinolate content in our collections ranged from $0.96 \mu \mathrm{mol} \mathrm{g}^{-1}$ dry weight (DW) to $46.02 \mu \mathrm{mol} \mathrm{g}^{-1} \mathrm{DW}$, with the aliphatic glucosinolates making up the highest ratio (75.5\%, Table S1). NAP was the most abundant glucosinolate, with a mean content of about $3.98 \mu \mathrm{mol} \mathrm{g}^{-1} \mathrm{DW}$, representing 30.5\% of the total glucosinolate content (Supplementary Table S4).

Among the five-candidate genes, $B r M A M-3$ (Bra013007) was significantly and positively associated with NAP $(0.70)$ and the accumulation of total glucosinolates $(0.57)(P<0.001)$ as well as $4 \mathrm{OH}(0.43)(P<0.01)$. BrMYB34.1 (Bra13000) was associated with NAP (0.42), $4 \mathrm{OH}(0.46)$, and the accumulation of total glucosinolates (0.40) $(P<0.01)$. BrMAM-5 (Bra013011) was associated with $4 \mathrm{OH}(0.44)$ and GBC $(0.47)(P<0.01)$. BrMYB28.1 (Bra012961) and BrMAM-4 (Bra013009) showed no correlation with glucosinolate accumulation. These results suggest that BrMAM-3 plays an important role in controlling the accumulation of aliphatic glucosinolates. Our inference agrees with the fact that overexpression of the AtMAM1 gene in Brassica spp. increases total aliphatic glucosinolate content ${ }^{42}$.

\section{Nucleotide polymorphisms of candidate genes situated within major QTLs}

We found that an insertion in $B r M A M-3$ that was related to the observed variations in aliphatic glucosinolates. The BrMAM-3 gene that was predicted consists of 11 exons, encoding 504 amino acids. The deduced protein sequence of the BrMAM-3 gene showed $81.2 \%$ and $77.9 \%$ identity with AtMAM1 and AtMAM3, respectively. The $B r M A M-3$ gene showed $100 \%$ identity with the L143 and Z16 accessions, except for one transposon insertion in exon 1. Furthermore, ten single-nucleotide substitutions (SNPs) across introns and exons were detected. Among the SNPs, three synonymous and one non-synonymous mutations were located in exons; the other SNPs were detected within introns, but did not affect the characteristics of amino acids. However, the BrMAM-3 transcript containing the $1.2-\mathrm{kb}$ transposon insertion fragment (Fig. 2) was predicted to be translated into a nonfunctional truncated protein of 101 amino acids that included a frame shift. We detected trace or no aliphatic glucosinolates in the Z16 accession, which harbors the $B r M A M-3$ allele with the transposon insertion.

No correlation with aliphatic glucosinolates was observed for the variations in BrMYB34.1, BrMYB28.1, $B r M A M-4$, and BrMAM-5. The gene sequences showed $100 \%$ identity between L143 and Z16 accessions, except for several SNPs across introns and exons. For example, there were two SNPs involved in BrMYB34.1, three in BrMYB28.1, and five in BrMAM-5 (Figure S1). These SNPs did not affect the characteristics of amino acids. Moreover, the BrMAM-4 and BrMAM-5 genes exhibited intact gene structures compared to AtMAM. The $B r M A M-4$ gene contains eight exons, and the BrMAM-5 gene has seven exons. These encode proteins with incomplete motifs, lacking two conserved motifs, respectively. The missing motifs belong to the PLN03228 (methylthioalkylmalate synthase) conserved domain, which may result in lower or inactive enzyme activity during methionine biosynthesis ${ }^{29}$. Therefore, the BrMYB34.1, BrMYB28.1, BrMAM-4, and BrMAM-5 genes were not considered key candidate genes that control the accumulation of aliphatic glucosinolates in B. rapa. $B r M A M-1$ and BrMAM-2, which are clustered as MF1, also had intact gene structures compared to AtMAM. $B r M A M-1$ lost one exon and $B r M A M-2$ encodes a protein that lacks two conserved motifs ${ }^{29}$. These two genes were not detected by QTL analysis, suggesting that although these have inherited the same MAM gene of $A$. thaliana, these did not inherit the key role in controlling aliphatic glucosinolates accumulation during rediploidization.

\section{Relationship between total aliphatic glucosinolates and nucleotide polymorphisms in BrMAM-3}

The insertion of $B r M A M-3$ was used to develop a PCR-based marker across exons 1 and 2 and was validated in Z16 and L143 accessions (Fig. 3). The marker was used to analyze the association between total aliphatic glucosinolate accumulation and the $B r M A M-3$ transposon-insertion alleles in a natural DH germplasm collection (Fig. 4). Correlation analysis showed that the 

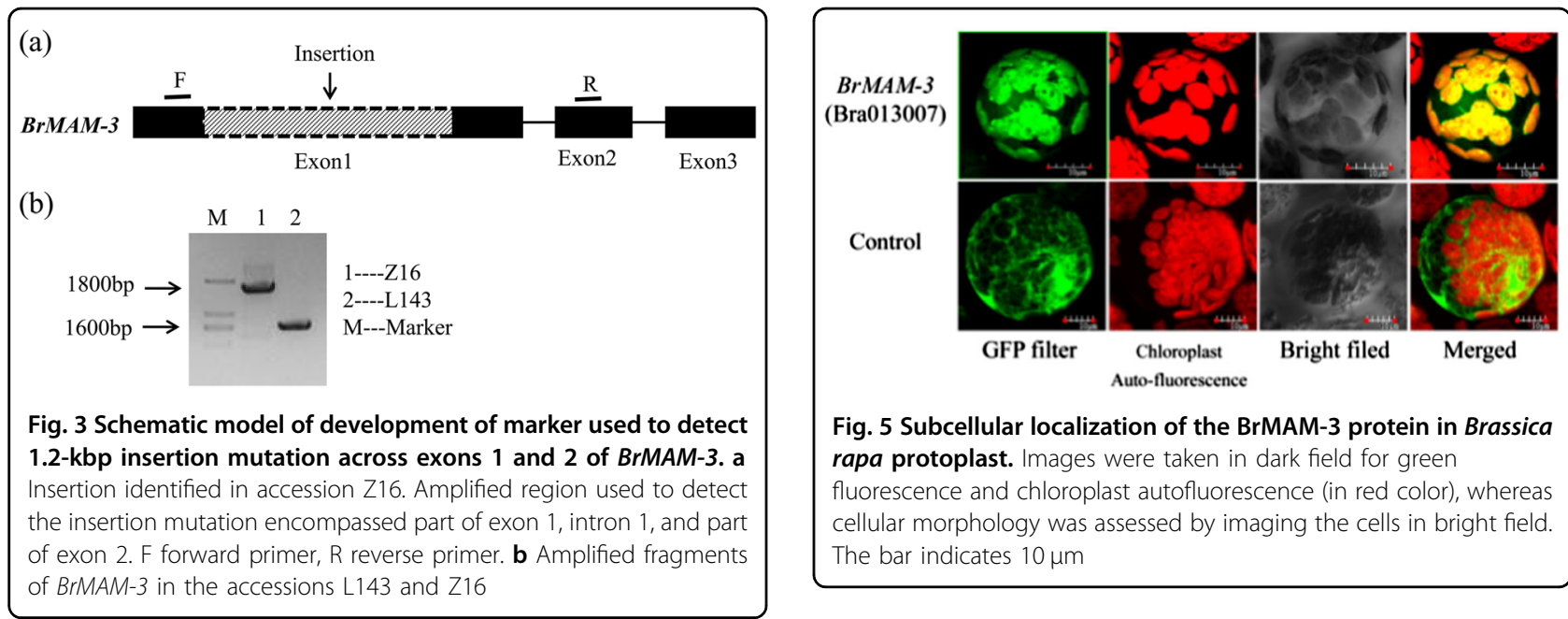

Fig. 5 Subcellular localization of the BrMAM-3 protein in Brassica rapa protoplast. Images were taken in dark field for green

fluorescence and chloroplast autofluorescence (in red color), whereas cellular morphology was assessed by imaging the cells in bright field. The bar indicates $10 \mu \mathrm{m}$

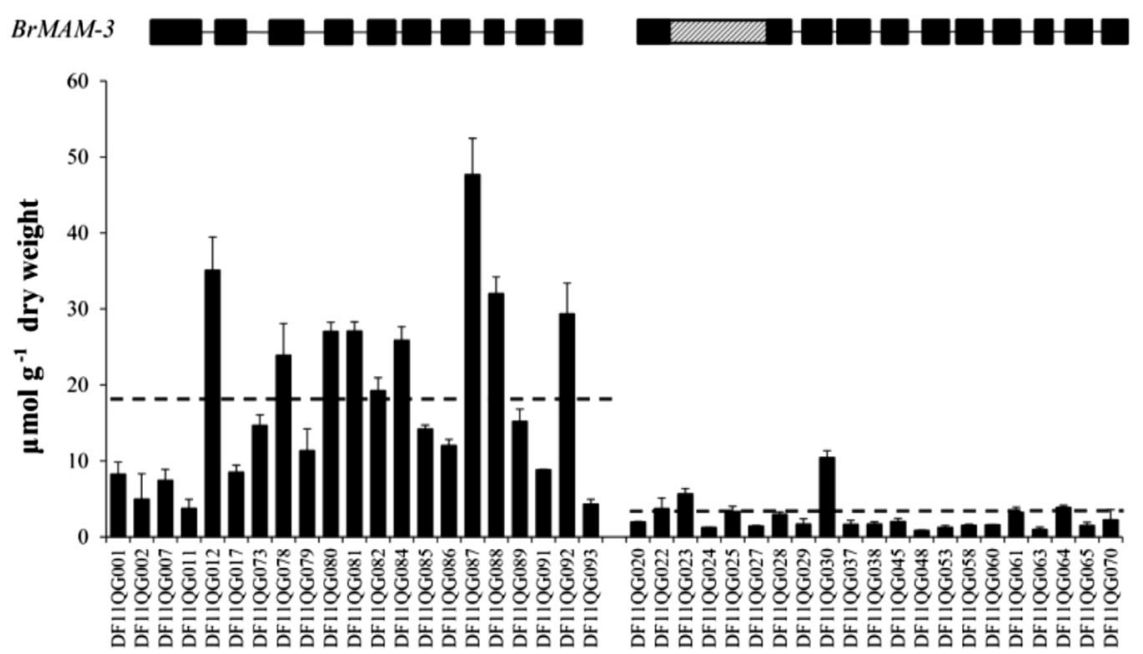

Fig. 4 Amplified fragments of $B r M A M-3$ and total aliphatic glucosinolates variations among germplasm collection of $B$. rapa leaves. Dashed lines indicate the average amount of total aliphatic glucosinolates

transposon-insertion polymorphism is significantly associated with variations in the total aliphatic glucosinolate accumulation among the tested $B$. rapa accessions. Of the 42 screened DH lines, half of the accessions possessed the functional allele, whereas the others harbored transposon insertions (Fig. 4). The accessions with the functional alleles had a significantly higher amount of total aliphatic glucosinolates than those with insertion alleles. The average number of total aliphatic glucosinolates in $\mathrm{DH}$ lines with the functional $B r M A M-3$ allele was about sixfold greater than those with the mutated insertion allele.

\section{BrMAM-3 is localized to the chloroplast}

We investigated the subcellular localization of $B r M A M-3$ to further analyze its characteristics. The Pro ${ }_{\text {CAMv35s: }}$ BrMAM-3:GFP vectors were constructed and detected by monitoring the transient expression of GFP in B. rapa mesophyll protoplast cells (Fig. 5). The transiently transformed cells showed strong green fluorescence signals in the chloroplasts, demonstrating that BrMAM-3 protein is a predominantly chloroplast-localized protein, which agrees with that reported of AtMAM3 in Arabidopsis (Col-0) ${ }^{13,16}$.

BrMAM-3 encodes a functional protein that controls the accumulation of aliphatic glucosinolates

A complementation test was performed by creating transgenic plants overexpressing the BrMAM-3 gene in the natural Arabidopsis mutant Landsberg erecta (Ler-0), in which the $5^{\prime}$ portion of $M A M 1$ is deleted ${ }^{12}$. Three independent homozygous lines of $\operatorname{BrMAM-3}$ were analyzed for total as well as individual glucosinolate fractions in 8-week-old rosette leaves. 


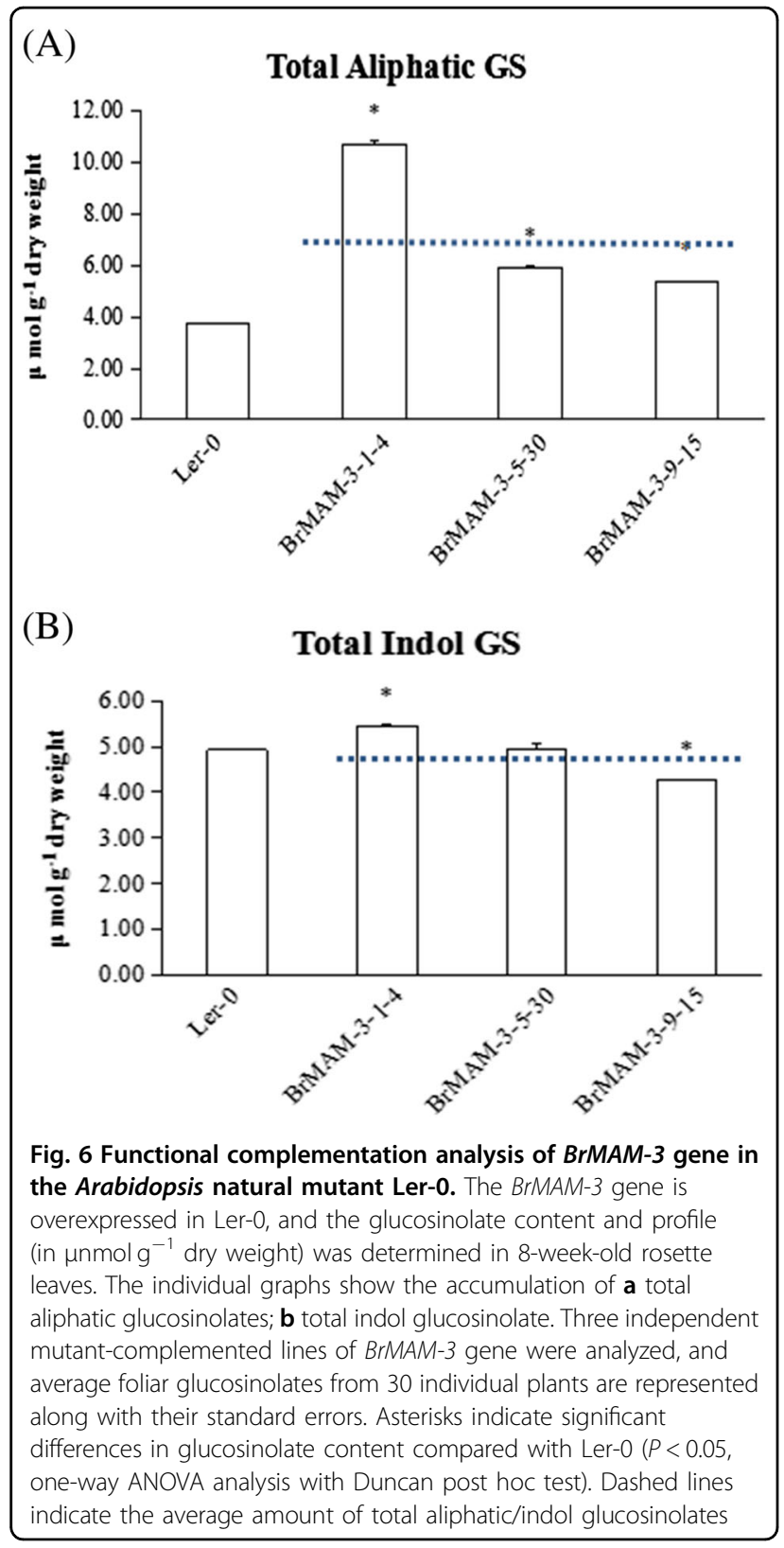

The functional complementation of the BrMAM-3 gene elevated the accumulation of total aliphatic glucosinolate 1.4- to 2.9-fold compared to Ler-0 (Fig. 6a). However, the average amount of total indol glucosinolates of the complement lines did not exceed that of Ler-0 (Fig. 6b). Thus, the results of mutant complementation analysis in $A$. thaliana suggest that the BrMAM-3 gene controls the levels of aliphatic glucosinolates.

\section{Expression patterns of BrMAM-3 are associated with the} accumulation of aliphatic glucosinolates in $B$. rapa leaves

We measured BrMAM-3 transcript expression in the accessions L143 and Z16 at the seedling and reproductive stages by qRT-PCR. The transcript levels of BrMAM-3

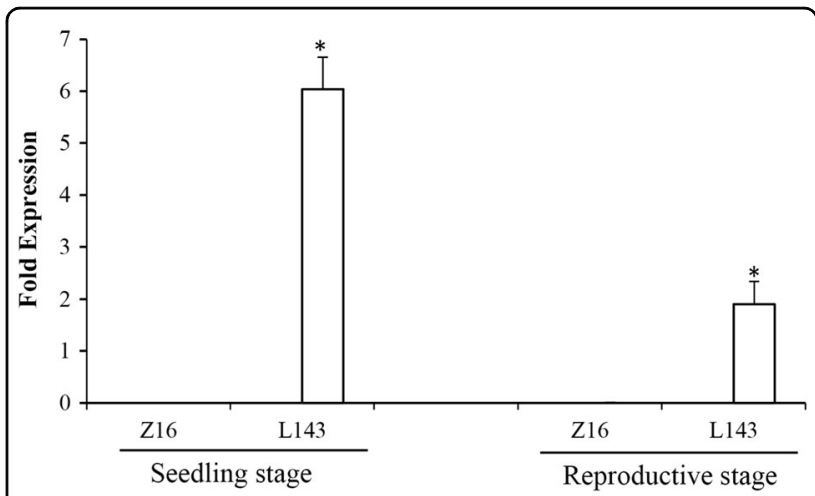

Fig. 7 Expression profile of BrMAM-3 gene at the seedling and reproductive stages in accessions Z16 and L143. Real-time

quantitative PCR (qRT-PCR) was conducted, and the expression levels of BrMAM-3 in L143 siliques are indicated. Error bars represent the standard deviation (SD) of three biological repeats. Asterisks indicate significant differences in the expression profile of the BrMAM-3 gene compared to the Z16 accession $(P<0.05$, determined by one-way ANOVA)

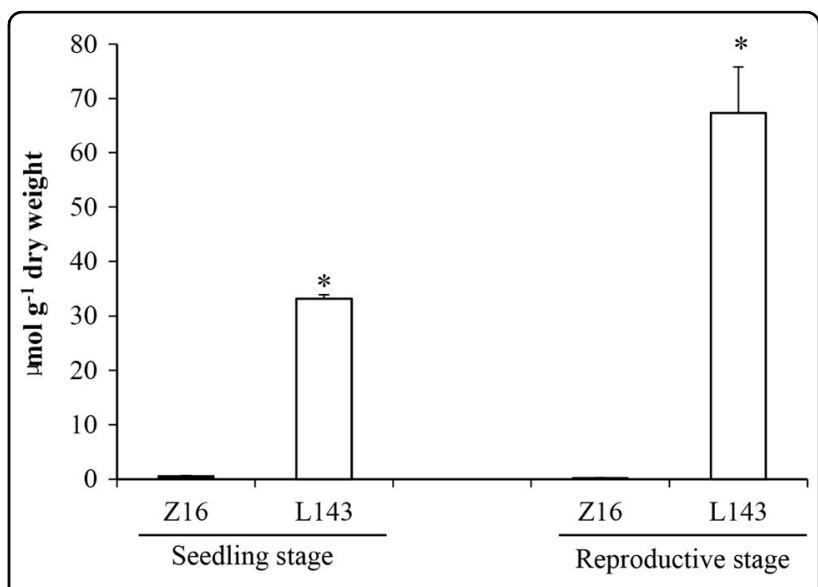

Fig. 8 Total aliphatic glucosinolates accumulation (in $\mu \mathrm{mol} \mathrm{g}^{-1}$ dry weight) in $\mathrm{Z16}$ and L143 at the seedling and reproductive stages. Error bars represent the standard deviation (SD) of three biological repeats. Asterisks indicate significant differences in aliphatic glucosinolates content compared to the Z16 accession $(P<0.05$, determined by one-way ANOVA)

were significantly higher in L143 than Z16 (Fig. 7). In Z16, undetectable or trace expression levels of $B r M A M-3$ were observed in both the seedling and reproductive stages. In contrast, BrMAM-3 was upregulated in glucosinolatesynthesizing tissues of L143 such as seedling leaves and mature leaves. The BrMAM-3 expression profile in L143 coincided with the pattern of AtMAM1 transcript accumulation, which showed maximum accumulation in expanding leaves followed by mature leaves. ${ }^{16}$

To test whether the transcription of BrMAM-3 gene contributes to the accumulation of total aliphatic glucosinolates in $B$. rapa, we analyzed the glucosinolate profiles 
of different developmental stages by high-performance liquid chromatography (HPLC). There were pronounced differences in the total aliphatic glucosinolate concentrations between Z16 and L143 both at the seedling and reproductive stages (Fig. 8). At the seedling stage, Z16 had trace amounts of total aliphatic glucosinolates in the leaves, whereas the expanding leaves of L143 depicted high aliphatic glucosinolate levels. These observations coincided with the expression pattern of BrMAM-3. In the reproductive stage, the total aliphatic glucosinolate in the leaves of L143 were higher than that of Z16. The total aliphatic glucosinolate accumulation in L143 at the seedling and reproductive stages was apparently inconsistent with the BrMAM-3 expression patterns. Therefore, other $B r M A M$ genes or the R2R3-BrMYB superfamily transcription factors may be involved in glucosinolate accumulation.

\section{Discussion}

Aliphatic glucosinolates, secondary metabolites known to be involved in plant defense, make up the majority of the glucosinolate content in A. thaliana and Brassica spp. In A thaliana, the amount of total aliphatic glucosinolates is regulated by three R2R3-MYB transcription factors (MYB28, MYB29, and MYB76), and their structural diversity arises from chain elongations of methionine and side-chain modifications. However, the molecular genetic mechanism controlling aliphatic glucosinolate accumulation in $B$. rapa is largely unknown. Our study implicates BrMAM-3 in controlling aliphatic glucosinolate accumulation. This research will pave the way for the genetic manipulation of total aliphatic glucosinolate levels in $B$. rapa.

\section{Variations in BrMAM-3 are related to reduced aliphatic glucosinolate levels in $B$. rapa}

Apart from the artificially generated genotypes and high-throughput technologies, the analysis of naturally occurring genetic variations provides insights into the control of important plant processes. Relationships between naturally occurring variants and aliphatic glucosinolates have been reported in $A$. thalian $a^{43-45}$ and $B$. olerace $^{46}$, but are limited in B. rapa and other Brassica species. A previous report has shown that silencing of the AtMAM gene family in B. napus canola and B. napus rapeseed reduces the content of aliphatic and total glucosinolates ${ }^{47}$.

We analyzed the association between the expression and structure of five-candidate genes and glucosinolate content and profiles to study the genes involved in the differential aliphatic glucosinolate accumulation among $B$. rapa accessions. Our results showed that the two $M Y B$ genes (BrMYB28.1 and BrMYB34.1) and two MAM genes (BrMAM-4 and BrMAM-5) showed no significant correlation with aliphatic glucosinolates, $B r M A M-3$ exhibited a significant positive correlation with aliphatic glucosinolates. Mutant complementation of $A$. thaliana showed that BrMAM-3 increases the amount of total aliphatic glucosinolates. However, detailed expression analysis using qRT-PCR assays revealed that $B r M A M-3$ has genotypic-specific expression partitioning in $B$. rapa. Moreover, a large insertion in exon 1 of $B r M A M-3$ among the accessions with a trace amount of total aliphatic glucosinolate indicates that the loss function of $B r M A M-3$ results in a reduction in total aliphatic glucosinolates in $B$. rapa. Meanwhile, the transposon insertion in the first exon of BrMAM-3 could lead to a different transcriptional regulation of BrMAM-3 between the L143 and Z16 accessions.

\section{Functional divergence could occur in the MAM of the major QTL}

Based on the results of phylogenetic and syntenic analyses of sequenced Brassicaceae species, we propose a lineage-specific evolution pattern for syntenic $M A M$ $\operatorname{loci}^{48}$. Upon divergence of the Brassica genus, B. rapa retained various copies of $M A M$ genes, which were generated from WGT, followed by biased gene loss.

The three tandem BrMAM genes in the major QTL locus, with the same gene order and orientation, are located in the conserved syntenic region of the LF. $B r M A M-4$ underwent a recent TD event to give rise to $B r M A M-5^{48}$. The present study showed that BrMAM-3 is responsible for the observed variations in aliphatic glucosinolates, indicating that $B r M A M-3$ retained the function of the $M A M a$ gene. Sequence analysis showed that BrMAM-4 and BrMAM-5 have intact gene structures compared to AtMAM, which lacks the methylthioalkylmalate synthase-conserved domain. Further investigation demonstrated that $B r M A M-4$ is not associated with the accumulation of glucosinolates, indicating that BrMAM-4 may have lost MAM enzyme activity. BrMAM5 is associated with $4 \mathrm{OH}$ and $\mathrm{GBC}$ but not aliphatic glucosinolates, suggesting that it acquired a new function (neofunctionalization) that is involved in indol glucosinoate biosynthesis.

BrMAM-1 and BrMAM-2 are located in the conserved syntenic region of the MF. BrMAM-1 underwent a recent TD event to give rise $B r M A M-2^{48}$. These two genes may have retained their catalytic activity for aliphatic glucosinoate accumulation, which requires validation in a future investigation.

\section{$B r M A M-3$ is a candidate for engineering the high aliphatic glucosinolate trait in $B$. rapa}

Glucosinolates have obtained the status of "model" secondary metabolites because their hydrolysis products exhibit different biological activities, e.g., as defense 
compounds and attractants. For humans, these compounds function as cancer-preventing agents, biopesticides, and flavor compounds. In the past decade, certain glucosinolates have been identified as potent cancerpreventing agents. Sulforaphane, the isothiocyanate derivative of glucoraphanin found in broccoli, has been the focus of many of these studies. These results aim to increase the sulforaphane content in broccoli and promote the health benefits of this vegetable. However, many Brassica species such as B. rapa, B. napus, and B. juncea harbor trace amounts of glucoraphanin, which is the precursor of sulforaphane ${ }^{49}$. RNA interference (RNAi) has been demonstrated to be an efficient method of silencing GSL-ELONG ${ }^{47}$ and GSL-ALK gene families ${ }^{50}$ to manipulate the beneficial glucosinolate profiles in B. napus. However, efforts in developing beneficial glucosinolate profiles in B. rapa are limited.

In $B$. rapa, the aliphatic glucosinolates are the predominant glucosinolates but vary among varieties in terms of content and glucosinolate profiles ${ }^{10,51}$. Our previous study identified three $B r A O P 2$ genes encoding the functional AOP2 that is involved in side-chain modifications of aliphatic glucosinolates ${ }^{29}$. Thus, it is possible to block $B r A O P 2$ genes to develop beneficial glucosinolates. In our current study, the accessions with functional $B r M A M-3$ alleles had a significantly higher amount of total aliphatic glucosinolates than those with insertion alleles, demonstrating that the $B r M A M-3$ gene plays an important role in controlling the accumulation of aliphatic glucosinolates in $B$. rapa. Thus, BrMAM-3 could be utilized to improve the amount of total aliphatic glucosinolates in B. rapa accessions with low aliphatic glucosinolate content. Silencing of $B r A O P 2$ genes and overexpression of the functional $B r M A M-3$ gene can be coupled to enrich the amount of beneficial glucosinolates in $B$. rapa.

Our findings provide functional evidence of expression partitioning of $B r M A M-3$ gene in controlling aliphatic glucosinolate content. Our results suggest that the naturally occurring transposon insertion in exon 1 of $B r M A M-3$ contributes largely to the observed variations in accumulation of total aliphatic glucosinolates in B. rapa. The information obtained in the current study may aid in manipulating the aliphatic glucosinolate content in Brassica crops using conventional breeding and/or transgenic approaches.

\section{Materials and methods Plant material}

To characterize the association of glucosinolate profiles of $B$. rapa and the expression pattern of five-candidate genes, we performed transcriptome profile sequencing in $52 \mathrm{~B}$. rapa accessions according to Cheng et al. ${ }^{52}$ (Supplementary Table S3). These accessions belong to 11 cultivar groups (Supplementary Table S5).
Two DH accessions Z16 and L143 were germinated and grown in a greenhouse at the Chinese Academy of Agricultural Sciences (Beijing, China) in the spring of 2011. Z16 is a Chinese cabbage accession with low glucosinolate content, whereas L143 is a yellow sarson accession with a high level of glucosinolates content. Leaf samples were collected from these plants for BrMAM-3 gene expression and glucosinolates profiling. Three biological replicates of each sample were prepared under normal growth conditions (10 weeks after sowing). Different tissues were collected, flash frozen in liquid nitrogen, and kept at $-80^{\circ} \mathrm{C}$ until further use.

Forty-two B. rapa accessions (Supplementary Table S5) were used to screen for BrMAM-3 sequence variations. The accessions were grown in a greenhouse in Beijing in the fall of 2011 to investigate aliphatic glucosinolate profiles. The temperature in the greenhouse was between 15 and $25^{\circ} \mathrm{C}$ night/day.

A. thaliana ecotype Landsberg erecta (Ler) was used for functional complementation in vivo. Seeds of Ler-0 were plated on soil and cold-treated at $4{ }^{\circ} \mathrm{C}$ for 3 days in the dark. After stratification, seeds were transferred into a temperature-controlled growth chamber under short-day conditions ( $8 \mathrm{~h}$ light, $16 \mathrm{~h}$ dark) at $21-24{ }^{\circ} \mathrm{C}$ and $40 \%$ humidity.

For plants grown on Petri dishes, the seeds were surface sterilized with $75 \%(\mathrm{v} / \mathrm{v})$ ethanol for $7 \mathrm{~min}$ and then washed thrice with sterile water. Seeds were sown on Murashige and Skoog (MS)-agar medium (one-halfstrength MS salt, $\mathrm{pH}$ 5.8) and cold-treated at $4{ }^{\circ} \mathrm{C}$ for 2 days in the dark, then placed in a growth chamber $(16 \mathrm{~h}$ of light at $22^{\circ} \mathrm{C}$ and $8 \mathrm{~h}$ of darkness at $18^{\circ} \mathrm{C}$ ). Transgenic plants were selected by germination on half-strength MS medium containing kanamycin/hygromycin antibiotics and were subsequently treated as wild-type plants.

\section{Development of $\mathrm{BC}_{2} \mathrm{DH}$ populations, QTL mapping, and isolation and sequencing of candidate genes}

The recurrent parents, L143 and L144 (with a high levels of glucosinolates), were used as the female and the donor as the male parent (Z16 accession) to generate the $F_{1}$ generation. A single $F_{1}$ plant (maternal) was backcrossed to the respective cultivars (paternal) to produce $\mathrm{BC}_{1} \mathrm{~F}_{1}$ plants. Then, each $\mathrm{BC}_{1} \mathrm{~F}_{1}$ plant was backcrossed a second time with the two cultivars. Two $\mathrm{BC}_{2} \mathrm{DH}$ populations designated as $\mathrm{RC}_{-} \mathrm{BC}_{2} \mathrm{DH}$ (derived from the cross L143) and $\mathrm{YS}_{-} \mathrm{BC}_{2} \mathrm{DH}$ (derived from the cross L144) were developed by another culture. A total of $250 \mathrm{BC}_{2} \mathrm{DH}$ lines were obtained from the two $\mathrm{BC}_{2} \mathrm{DH}$ populations.

For the analysis of phenotypic measurement of aliphatic glucosinolates, 120 individual lines of each $\mathrm{BC}_{2} \mathrm{DH}$ populations were used and grown in the greenhouse in two different seasons. Linkage maps were constructed using JoinMap 4 and QTL analysis was performed with 
MapQTL4 (https://www.kyazma.nl/). QTL mapping was initially performed on transformed data with interval mapping (IM) followed by composite interval mapping, referred to as MQM mapping in MapQTL4. The significant cofactors for each MQM model were determined through an iterative automatic cofactor selection. The genome-wide logarithm of odds (LOD) significance threshold was obtained from permutation tests with 1000 replicates as implemented in MapQTL4.

To detect possible variations in the candidate genes involved on the major QTL locus in L143 and Z16 accessions, the candidate gene sequences were amplified with specific primers (Supplementary Table S6) from genomic DNA, and sequenced using an ABI3730XL analyzer and analyzed using ClustalX.

\section{Generation of transgenic plants}

The coding sequences of $B r M A M-3$ gene were isolated and amplified using L143 cDNA as template with the gene-specific primers, including restriction sites (BrMAM-3, forward primer with $K p n I$ restriction site: 5'GGGGTACCATGGCTTCGTCACTTCTG-3', reverse primer with $\mathrm{XbaI}$ restriction site: 5'-GCTCTAGATTA TACCACAGAAGAAATC-3'), and ligated to a pEASYT1 vector. Following sequence analysis, the pEASY-T1: $B r M A M-3$ constructs were digested with $\mathrm{KpnI} / \mathrm{Xba \textrm {I }}$ and inserted into the pCambia 1300 vector driven by a CaMV35S promoter. The resulting construct was verified by DNA sequencing and subsequently transformed into Agrobacterium tumefaciens (strain GV3101). The binary vector pCambia 1300 containing a hygromycin resistance gene was utilized in the selection of transformed Arabidopsis lines. The floral infiltration method ${ }^{53,54}$ was used to transform natural mutant Ler-0 plants. Transgenic plants were selected by germination on half-strength MS medium containing $30 \mu \mathrm{g} \mathrm{mL}^{-1}$ hygromycin antibiotics and were subsequently treated as wild-type plants. The T2 generation derived from the selected plants was used to identify homozygous transformed lines. The T3 generation homozygous plants were subsequently employed in HPLC analysis.

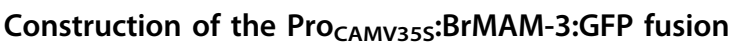 plasmid and transformation of $B$. rapa mesophyll protoplast cells}

To identify the subcellular localization of the BrMAM-3

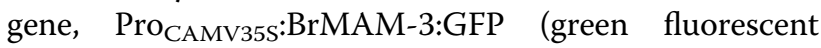
protein) constructs were generated. The BrMAM-3 coding sequences without stop codon were isolated and amplified from cDNA with the gene-specific primers including restriction sites (forward primer with an $\mathrm{Xba \textrm {I }}$ restriction site: 5'-TCTAGAATGGCTTCGTCACTTCT GAC-3', reverse primer with KpnI restriction site: $5^{\prime}$-TTGGTACC TACCACAGAAGAAATC-3'). The
PCR-amplified BrMAM-3 was inserted into the pSPYCE-

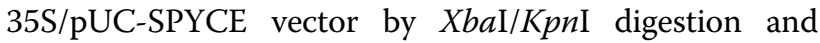
ligation. The resulting constructs were verified by DNA sequencing. The subcellular locations of the BrMAM-3 genes were detected by monitoring the transient expression of GFP in B. rapa mesophyll protoplast cells ${ }^{54}$ on an OLYMPUS FV1200 Laser Confocal System. GFP fluorescence was imaged at an excitation wavelength of 488 $\mathrm{nm}$, and the emission signal was detected between 495 and $530 \mathrm{~nm}$ for GFP and between 643 and $730 \mathrm{~nm}$ for chlorophyll autofluorescence.

\section{Glucosinolate extraction and HPLC analysis}

The extraction and quantification of glucosinolates were performed by HPLC as previously described (Hehongju protocols, 2002). Lyophilized samples $(0.2 \mathrm{~g})$ were weighed in $15-\mathrm{mL}$ plastic tubes and immersed in boiling methyl alcohol $(5 \mathrm{~mL})$ containing $100 \mu \mathrm{L}$ benzyl glucosinolate as the internal standard. After $20 \mathrm{~min}$ of gentle shaking, samples were cooled at $4{ }^{\circ} \mathrm{C}$ and centrifuged at $3000 \times g$ for $10 \mathrm{~min}$. The supernatant (extract) was cleaned twice with 70\% methyl alcohol. The extracts were loaded onto DEAE Sephadex A25 columns and desulfated overnight using purified sulfatase before HPLC. The column was then washed thrice with $0.5 \mathrm{~mL}$ deionized water, and the eluent that was filtered using a $0.45 \mu \mathrm{m}$ membrane was used for HPLC analysis. Specific glucosinolates were identified by comparing retention times and UV absorption spectra with purified standards. Concentrations of individual glucosinolates were calculated as nmol $\mathrm{mg}^{-1}$ DW relative to the area of the internal standard peak using the respective response factors reported earlier ${ }^{55}$.

\section{Reverse transcriptase-mediated first-strand synthesis and real-time RT-PCR analysis}

Total RNA was isolated from different organs of accessions L143 and Z16 using a total RNA extraction kit according to the manufacturer's instructions (Sangon, http://www.sangon.com) and then treated with DNase I (Sigma-Aldrich) to eliminate any DNA contamination. RNA purity was determined spectrophotometrically, and quality was determined by examining rRNA bands on $1 \%$ agarose gels. cDNAs were synthesized from $\sim 2 \mu \mathrm{g}$ of total RNA using TransScript First-Strand cDNA Synthesis SuperMix (Transgen, www.transgen.com.cn) with oligo $(\mathrm{dT})$ as primer in a $20-\mu \mathrm{L}$ reaction.

The specificity of the primers of $B r M A M-3$ and $B r G A P D H$ was verified by DNA sequencing after their PCR products were cloned into $\mathrm{pEASY}-\mathrm{T} 1$ vectors. The efficiency of gene-specific BrMAM-3 and GAPDH primer pairs was initially ascertained using a fourfold serial dilution of the L143 cDNA. A linear correlation coefficient $\left(R^{2}\right)$ of 0.95 and above was observed over a 64 -fold 
dilution range, which reflected the high efficiency of each primer pair. Real-time quantitative PCR (RT-qPCR) was performed in a total volume of $15 \mu \mathrm{L}$, which included $2 \mu \mathrm{L}$ of diluted cDNA, $0.5 \mu \mathrm{L}$ of each primer $(10 \mathrm{pM})$, and $7.5 \mu \mathrm{L}$ of $2 \times$ SYBR Green Master Mixes (Thermo Fisher, USA) on an Eppendorf real-Time PCR system (Eppendorf, Germany), according to the kit manual. The RTqPCR program was conducted at $95^{\circ} \mathrm{C}$ for $2 \mathrm{~min}$, followed by 40 cycles of $95^{\circ} \mathrm{C}$ for $30 \mathrm{~s}$ and $60^{\circ} \mathrm{C}$ for $60 \mathrm{~s}$. The expression level of $B r G A P D H$ was used as an internal control, and the expression of other genes was computed using the $2^{-\Delta \Delta C T}$ method $^{56}$. The primers used in this work are listed in Supplementary Table S7.

\section{Insertion marker analysis}

The forward primer $\mathrm{F}$ (5'-CGTCCGTACAA CAAGTCATCC- $3^{\prime}$ ) in exon 1 and the reverse primer $\mathrm{R}$ (5'-AACTTAACACTACTCGCGGCC-3') in exon 2 were designed to develop an insertion marker for BrMAM-3. PCR was performed under the following conditions: denaturation at $94{ }^{\circ} \mathrm{C}$ for $5 \mathrm{~min}$, followed by 30 cycles of amplification $\left(94{ }^{\circ} \mathrm{C}\right.$ for $45 \mathrm{~s}, 55^{\circ} \mathrm{C}$ for $45 \mathrm{~s}$, and $72{ }^{\circ} \mathrm{C}$ for $1.5 \mathrm{~min}$ ), and a final extension at $72^{\circ} \mathrm{C}$ for $10 \mathrm{~min}$. The PCR products were separated on a $1 \%$ agarose gel to determine the genotype of the insertion marker.

\section{Statistical analysis}

Data were analyzed for statistical significance using oneway ANOVA with Duncan's post hoc test using the SPSS software. A $P$ value $<0.05$ was considered as significant.

\section{Acknowledgements}

The National Key Research and Development Program of China (equally by 2016YFD0100307 and 2016YFD0100506), the National Natural Science Foundation of China (31630068) and Application Basic Research Program of Qingdao [14-2-4-112-jch] supported this study. The work was also funded by the Science and Technology Innovation Program of the Chinese Academy of Agricultural Sciences. This research work was carried out in the Key Laboratory of Biology and Genetic Improvement of Horticultural Crops, Ministry of Agriculture, China.

\section{Author details}

${ }^{1}$ Institute of Vegetables and Flowers, Chinese Academy of Agricultural Sciences, 100081 Beijing, China. ${ }^{2}$ Institute of Southern Economic Crops, Institute of Bast Fiber Crops, Chinese Academy of Agricultural Sciences, 410205 Changsha, China. ${ }^{3}$ College of Horticulture, Qingdao Agricultural University, 266109 Qingdao, China

\section{Conflict of interest}

The authors declare that they have no conflict of interest.

\section{Publisher's note}

Springer Nature remains neutral with regard to jurisdictional claims in published maps and institutional affiliations.

Supplementary Information accompanies this paper at (https://doi.org/ 10.1038/s41438-018-0074-6).
Received: 17 January 2018 Revised: 28 May 2018 Accepted: 5 July 2018 Published online: 01 December 2018

\section{References}

1. Rask, L. et al. Myrosinase: gene family evolution and herbivore defense in Brassicaceae. Plant Mol. Biol. 42, 93-113 (2000).

2. Fahey, J. W. et al. Sulforaphane inhibits extracellular, intracellular, and antibiotic-resistant strains of Helicobacter pylori and prevents benzo[a]pyreneinduced stomach tumors. Proc. Natl Acad. Sci. USA 99, 7610-7615 (2002).

3. Fahey JW, Z. Y. \& Talalay, P. Broccoli sprouts: an exceptionally rich source of inducers of enzymes that protect against chemical carcinogens. Proc. Natl Acad. Sci. USA 94, 10367-10372 (1997).

4. Gamet-Payrastre, L. et al. Phytochemicals from cruciferous plants protect against cancer by modulating carcinogen metabolism. J. Nutr. 131 3027-3033 (2001)

5. Traka, M. \& Mithen, R. Glucosinolates, isothiocyanates and human health. Phytochem. Rev. 8, 269-282 (2009).

6. Zhang, Y., Kensler, T. W., Cho, C. G., Posner, G. H. \& Talalay, P. Anticarcinogenic activities of sulforaphane and structurally related synthetic norbornyl isothiocyanates. Proc. Natl Acad. Sci. USA 91, 3147-3150 (1994).

7. Faulkner, K., Mithen, R. \& Williamson, G. Selective increase of the potential anticarcinogen 4-methylsulphinylbutyl glucosinolate in broccoli. Carcinogenesis 19, 605-609 (1998).

8. Clay, N. K., Adio, A. M., Denoux, C., Jander, G. \& Ausubel, F. M. Glucosinolate metabolites required for an Arabidopsis innate immune response. Science 323, 95-101 (2009).

9. Kroymann, J., Donnerhacke, S., Schnabelrauch, D. \& Mitchell-Olds, T. Evolutionary dynamics of an Arabidopsis insect resistance quantitative trait locus. Proc. Natl Acad. Sci. USA 100, 14587-14592 (2003). Suppl 2.

10. Padilla, G., Cartea, M. E., Velasco, P., de Haro, A. \& Ordas, A. Variation of glucosinolates in vegetable crops of Brassica rapa. Phytochemistry $\mathbf{6 8}, 536-545$ (2007).

11. Schonhof, I., Krumbein, A. \& Bruckner, B. Genotypic effects on glucosinolates and sensory properties of broccoli and cauliflower. Nahrung 48, 25-33 (2004).

12. Benderoth, M. et al. Positive selection driving diversification in plant secondary metabolism. Proc. Natl Acad. Sci. USA 103, 9118-9123 (2006).

13. Sonderby, I. E., Geu-Flores, F. \& Halkier, B. A. Biosynthesis of glucosinolatesgene discovery and beyond. Trends Plant. Sci. 15, 283-290 (2010).

14. Kliebenstein, D. J., Lambrix, V. M., Reichelt, M., Gershenzon, J. \& Mitchell-Olds, T. Gene duplication in the diversification of secondary metabolism: Tandem 2oxoglutarate-dependent dioxygenases control glucosinolate biosynthesis in Arabidopsis. Plant Cell 13, 681-693 (2001)

15. Textor, S. et al. Biosynthesis of methionine-derived glucosinolates in Arabidopsis thaliana: recombinant expression and characterization of methylthioalkylmalate synthase, the condensing enzyme of the chain-elongation cycle. Planta 218, 1026-1035 (2004).

16. Textor, S., de Kraker, J. W., Hause, B., Gershenzon, J. \& Tokuhisa, J. G. MAM3 catalyzes the formation of all aliphatic glucosinolate chain lengths in Arabidopsis. Plant Physiol. 144, 60-71 (2007).

17. De Kraker, J. W. \& Gershenzon, J. From amino acid to glucosinolate biosynthesis: protein sequence changes in the evolution of methylthioalkylmalate synthase in Arabidopsis. Plant Cell 23, 38-53 (2011).

18. Beekwilder, J. et al. The impact of the absence of aliphatic glucosinolates on insect herbivory in Arabidopsis. PLoS ONE 3, e2068 (2008).

19. Gigolashvili, T., Engqvist, M., Yatusevich, R., Muller, C. \& Flugge, U. I. HAG2/ MYB76 and HAG3/MYB29 exert a specific and coordinated control on the regulation of aliphatic glucosinolate biosynthesis in Arabidopsis thaliana. New Phytol. 177, 627-642 (2008).

20. Gigolashvili, T., Yatusevich, R., Berger, B., Muller, C. \& Flugge, U. I. The R2R3-MYB transcription factor HAG1/MYB28 is a regulator of methionine-derived glucosinolate biosynthesis in Arabidopsis thaliana. Plant J. 51, 247-261 (2007).

21. Hirai, M. Y. et al. Omics-based identification of Arabidopsis Myb transcription factors regulating aliphatic glucosinolate biosynthesis. Proc. Natl Acad. Sci. USA 104, 6478-6483 (2007).

22. Sonderby, I. E. et al. A systems biology approach identifies a R2R3 MYB gene subfamily with distinct and overlapping functions in regulation of aliphatic glucosinolates. PLOS ONE 2, e1322 (2007).

23. Gigolashvili, T. et al. The transcription factor HIG1/MYB51 regulates indolic glucosinolate biosynthesis in Arabidopsis thaliana. Plant J. 50, 886-901 (2007). 
24. Schweizer, F. et al. Arabidopsis basic helix-loop-helix transcription factors MYC2, MYC3, and MYC4 regulate glucosinolate biosynthesis, insect performance, and feeding behavior. Plant Cell 25, 3117-3132 (2013).

25. Takuno, S. et al. Effects of recombination on hitchhiking diversity in the Brassica self-incompatibility locus complex. Genetics 177, 949-958 (2007).

26. Lou, P. et al. Quantitative trait loci for glucosinolate accumulation in Brassica rapa leaves. New Phytol. 179, 1017-1032 (2008).

27. Wang, $H$. et al. Glucosinolate biosynthetic genes in Brassica rapa. Gene 487, 135-142 (2011).

28. Kim, Y. B. et al. MYB transcription factors regulate glucosinolate biosynthesis in different organs of Chinese cabbage (Brassica rapa ssp. pekinensis). Molecules 18, 8682-8695 (2013).

29. Zhang, J. et al. Three genes encoding AOP2, a protein involved in aliphatic glucosinolate biosynthesis, are differentially expressed in Brassica rapa. J. Exp. Bot. 66, 6205-6218 (2015).

30. Mithen, R., Clarke, J., Lister, C. \& Dean, C. Genetics of aliphatic glucosinolates. III. Side-chain structure of aliphatic glucosinolates in Arabidopsis thaliana. Heredity 74, 210-215 (1995).

31. Koornneef, M., Alonso-Blanco, C. \& Vreugdenhil, D. Naturally occurring genetic variation in Arabidopsis thaliana. Annu. Rev. Plant Biol. 55, 141-172 (2004).

32. Kliebenstein, D. J., Pedersen, D., Barker, B. \& Mitchell-Olds, T. Comparative analysis of quantitative trait loci controlling glucosinolates, myrosinase and insect resistance in Arabidopsis thaliana. Genetics 161, 325-332 (2002).

33. Pfalz, M., Vogel, H., Mitchell-Olds, T. \& Kroymann, J. Mapping of QTL for resistance against the crucifer specialist herbivore Pieris brassicae in a new Arabidopsis inbred line population, Da(1)-12 x Ei-2. PLoS ONE 2, e578 (2007).

34. Giamoustaris, A. \& Mithen, R. Genetics of aliphatic glucosinolates. IV. Side-chain modification in Brassica oleracea. Theor. Appl. Genet. 93, 1006-1010 (1996).

35. Howell, P. M., Sharpe, A. G. \& Lydiate, D. J. Homoeologous loci control the accumulation of seed glucosinolates in oilseed rape (Brassica napus). Genome 46, 454-460 (2003)

36. Uzunova, M., Ecke, W., Weissleder, K. \& Robbelen, G. Mapping the genome of rapeseed (Brassica napus L.). I. Construction of an RFLP linkage map and localization of QTLs for seed glucosinolate content. Theor. Appl. Genet. 90, 194-204 (1995).

37. Zhao, J. \& Meng, J. Detection of loci controlling seed glucosinolate content and their association with Sclerotinia resistance in Brassica napus. Plant Breed. 122, 19-23 (2003).

38. Mahmood, T., Ekuere, U., Yeh, F., Good, A. G. \& Stringam, G. R. Molecular mapping of seed aliphatic glucosinolates in Brassica juncea. Genome $\mathbf{4 6}$ 753-760 (2003).

39. Ramchiary, N. et al. QTL analysis reveals context-dependent loci for seed glucosinolate trait in the oilseed Brassica juncea: importance of recurrent selection backcross scheme for the identification of 'true' QTL. Theor. Appl. Genet. 116, 77-85 (2007).
40. Hittinger, C. T. \& Carroll, S. B. Gene duplication and the adaptive evolution of a classic genetic switch. Nature 449, 677-671 (2007).

41. Franzke, A., Lysak, M. A., Al-Shehbaz, I. A., Koch, M. A. \& Mummenhoff, K. Cabbage family affairs: the evolutionary history of Brassicaceae. Trends Plant Sci. 16, 108-116 (2011).

42. Zang, Y. X., Kim, J. H., Park, Y. D., Kim, D. H. \& Hong, S. B. Metabolic engineering of aliphatic glucosinolates in Chinese cabbage plants expressing Arabidopsis MAM1, CYP79F1, and CYP83A1. BMB Rep. 41, 472-478 (2008).

43. Kliebenstein, D. J. et al. Genetic control of natural variation in Arabidopsis glucosinolate accumulation. Plant Physiol. 126, 811-825 (2001).

44. Mithen, R., Raybould, A. F. \& Giamoustaris, A. Divergent selection for secondary metabolites between wild populations of Brassica oleracea and its implications for plant-herbivore interactions. Heredity 5, 472-484 (1995).

45. Neal, C. S., Fredericks, D. P., Griffiths, C. A. \& Neale, A. D. The characterisation of AOP2: a gene associated with the biosynthesis of aliphatic alkenyl glucosinolates in Arabidopsis thaliana. BMC Plant Biol. 10, 170 (2010).

46. Li G, QuirosC. F. In planta side-chain glucosinolate modification in Arabidopsis by introduction of dioxygenase Brassica homolog BoGSL-ALK. Theor. Appl. Genet. 106, 1116-1121 (2003).

47. Liu, Z. et al. MAM gene silencing leads to the induction of C3 and reduction of C4 and C5 side-chain aliphatic glucosinolates in Brassica napus. Mol. Breed. 27, 467-478 (2010).

48. Zhang, J. et al. Lineage-specific evolution of methylthioalkylmalate synthases (MAMs) involved in glucosinolates biosynthesis. Front. Plant Sci. 6, 18 (2015).

49. Rosa, E. A. S., Heaney, R. K., Fenwick, G. R. \& Portas, C. A. M. Glucosinolates in crop plants. Hortic. Rev. 19, 99-215 (1997).

50. Liu, Z. et al. Reducing progoitrin and enriching glucoraphanin in Brassica napus seeds through silencing of the GSL-ALK gene family. Plant Mol. Biol. 79, 179-189 (2012)

51. Kim, J. K. et al. Variation of glucosinolates in vegetable crops of Brassica rapa $\mathrm{L}$. ssp. pekinensis. Food Chem. 119, 423-428 (2010).

52. Cheng, F. et al. Subgenome parallel selection is associated with morphotype diversification and convergent crop domestication in Brassica rapa and Brassica oleracea. Nat. Genet. 48, 1218-1224 (2016).

53. Clough, S. J. \& Bent, A. F. Floral dip: a simplified method for Agrobacteriummediated transformation of Arabidopsis thaliana. Plant J. 16, 735-743 (1998).

54. Yoo, S. D., Cho, Y. H. \& Sheen, J. Arabidopsis mesophyll protoplasts: a versatile cell system for transient gene expression analysis. Nat. Protoc. 2, 1565-1572 (2007).

55. Brown, P. D., Tokuhisa, J. G., Reichelt, M. \& Gershenzon, J. Variation of glucosinolate accumulation among different organs and developmental stages of Arabidopsis thaliana. Phytochemistry 62, 471-481 (2003).

56. Livak, K. J. \& Schmittgen, T. D. Analysis of relative gene expression data using real-time quantitative PCR and the 2(-Delta Delta C(T)) method. Methods $\mathbf{2 5}$, 402-408 (2001) 